\title{
Profession médicale et gouvernement : l'abîme se creuse
}

Les opinions exprimées dans cet éditorial sont celles de l'auteur et ne représentent pas nécessairement celles de l'éditeur.

1 semble être de plus en plus difficile de pratiquer la médecine au Canada. On dirait que la difficulté s'accentue chaque mois, et ce n'est pas normal. Généralement, les médecins canadiens se sont montrés plutôt confiants à l'égard de la surveillance et du rôle exercés par le gouvernement dans la gestion des soins de santé. En fait, notre système en dépend; c'est pourquoi nous avons misé sur la communication bidirectionnelle pour en assurer le fonctionnement. Dans ce cas, pourquoi l'écart s'élargit-il entre les besoins des médecins en matière de soins aux patients et les budgets prévus par les ministères de la Santé?

La communication s'est indéniablement détériorée en Ontario. On pourrait croire qu'avoir un médecin comme ministre de la Santé faciliterait les échanges, mais il semble que ce n'ait pas été le cas ici. Le manque de leadership de l'Association médicale de l'Ontario (AMO) a permis au gouvernement d'ignorer les commentaires des médecins pour mettre de l'avant un plan qui ne ferait que nuire aux patients. La décision a été annulée à la dernière minute grâce à un groupe de médecins, la Coalition of Ontario Doctors, qui a fait ce que la direction de l'AMO aurait dû faire : garantir la prestation de soins aux patients. Eric Hoskins, ministre de la Santé de l'Ontario - l'une des 3 provinces où cette fonction est assurée par un médecin -, avait prévu geler le budget alloué à la santé malgré le vieillissement de la population, la croissance démographique et l'accroissement des besoins. Il s'attendait à ce que le système, déjà incapable de fournir des soins adéquats à la population actuelle, poursuive ses activités sans aucun financement supplémentaire. Ce modèle ne correspond pas vraiment à la vision proactive dont le pays a besoin en ce moment, et je doute que ce jeu en vaille la chandelle pour le Ministère. Tandis que les médecins se préoccupent des soins aux patients, le gouvernement ne pense qu'à geler les dépenses en santé pour équilibrer son budget. En outre, le gouvernement en place s'est montré incapable de gérer les dossiers des infrastructures, de l'électricité, de la croissance économique et du régime de retraite de l'Ontario. Et n'oublions pas le scandale d'Action Cancer Ontario, la Contribution-santé et les problèmes chez cyberSanté, qui nous donnent peu d'espoir de voir le gouvernement faire mieux en matière de santé.

L'Ontario est-il seul dans sa situation? Probablement pas. Mais comme plus du tiers des médecins canadiens exercent dans cette province, nous sommes tous touchés par ce qui s'y passe. Le fait que $80 \%$ des médecins de la Nouvelle-Ecosse aient voté pour une entente qui sera probablement insuffisante pour compenser l'augmentation du coût de la vie est un autre exemple de la méfiance accrue des médecins à l'égard du processus de négociations.

La surveillance et l'ingérence de la part du gouvernement fédéral n'amélioreront ni ce processus ni les soins aux patients. La santé est une compétence provinciale, pour le meilleur et pour le pire; le fédéral ne devrait donc pas avoir le champ libre s'il tente d'augmenter ses appuis politiques en s'appropriant des programmes particuliers, comme les soins à domicile. Je trouve extrêmement troublantes les rumeurs selon lesquelles Ottawa menacerait de ne plus verser de paiements de transfert si le secteur privé venait à s'étendre davantage. Il est évident, pour quiconque défend les soins de santé, que la privatisation de certains services est essentielle à la survie du système public. Les provinces doivent prendre leurs décisions en faisant preuve de prévoyance et en ayant une connaissance approfondie des réalités locales des soins de santé. Ces décisions ne devraient pas être influencées par un gouvernement fédéral déconnecté, qui ne connaît plus le contexte clinique depuis des dizaines d'années. À ce sujet, j'ai hâte que soit entendue l'affaire Brian Day en Colombie-Britannique, qui est constamment repoussée (par le gouvernement). Dans cette affaire, la reconnaissance juridique apparemment évidente du danger des règles en vigueur pour la santé des patients devrait ouvrir la porte à une reconfiguration des systèmes de santé visant à les adapter à la situation actuelle.

De façon générale, les signes que le système de santé n'est pas en santé lui-même ne manquent pas. Les médecins qui donnent des soins ont été mis de côté par la prise de décisions unilatérales à tous les paliers de gouvernement. De plus, la population est récemment devenue plus critique à l'endroit des médecins : elle semble les blâmer d'offrir un piètre accès aux soins malgré leurs salaires élevés. Les médecins ontariens peuvent au moins compter sur un sous-groupe actif qui travaille à améliorer le processus décisionnel dans le domaine des soins; toutefois, c'est l'ensemble du pays qui devrait s'inquiéter des décisions administratives répressives. Il est maintenant temps pour la profession médicale de reprendre la place qui lui revient, aux côtés du gouvernement, dans la prise de décisions en matière de soins au pays.

\section{Edward J. Harvey, MD \\ Corédacteur, fournal canadien de chirurgie}

Intérêts concurrents: E.J. Harvey est médecin hygiéniste en chef de Greybox Healthcare (Montréal) et président du Conseil d'administration de NXT-Sens Inc. (Montréal).

DOI: $10.1503 /$ cjs. 012316 